Relations industrielles

Industrial Relations

\title{
Linder, Marc, Protecting Capitalism: A History of the Internationalization of the Construction Industry
}

\section{Jean Sexton}

Volume 50, numéro 1, 1995

URI : https://id.erudit.org/iderudit/051008ar

DOI : https://doi.org/10.7202/051008ar

Aller au sommaire du numéro

Éditeur(s)

Département des relations industrielles de l'Université Laval

ISSN

0034-379X (imprimé)

1703-8138 (numérique)

Découvrir la revue

Citer ce compte rendu

Sexton, J. (1995). Compte rendu de [Linder, Marc, Protecting Capitalism: A History of the Internationalization of the Construction Industry]. Relations industrielles / Industrial Relations, 50(1), 242-243.

https://doi.org/10.7202/051008ar

Tous droits réservés (C) Département des relations industrielles de l'Université Laval, 1995
Ce document est protégé par la loi sur le droit d'auteur. L’utilisation des services d'Érudit (y compris la reproduction) est assujettie à sa politique d'utilisation que vous pouvez consulter en ligne.

https://apropos.erudit.org/fr/usagers/politique-dutilisation/ 
l'objectif opérationnel du volume et sa structure ont su éviter la fragmentation du sujet. Les chapitres, de longueur (une vingtaine de pages), de discours et de structures passablement uniformes, présentent donc la matière de manière utile à l'initié et accessible pour le noninitié. Le volume est aussi très facile à consulter grâce aux deux index (par sujets et par auteurs).

Que retirer en définitive de cet ouvrage sinon un enseignement sur la construction d'un discours multidisciplinaire. Il faut donc lui reconnaître deux mérites: la richesse et la variété théorique des articles si bien intégrés ainsi que la finesse des analyses et critiques présentées. Ce livre constitue pour les chercheurs, les étudiants engagés et les gestionnaires une source de réflexion susceptible de les aider à mieux comprendre la façon dont les femmes gèrent le stress lié au travail.

ÉRICK CHAMBERLAND Université Laval

\section{Projecting Capitalism: A History of the Internationalization of the Construction Industry}

by Marc Linder, Westport, Connecticut, Greenwood Press, 1994, 270 p., ISBN 0-313-29293-0 and ISSN 0084-9235.

Voici un ouvrage qui aborde l'industrie de la construction d'une façon originale et fort instructive. En utilisant une approche historique, l'auteur tente de détruire le mythe que la construction est une industrie locale regroupant plusieurs petites entreprises en parfaite concurrence et à l'abri des marchés mondiaux.

Pour étayer sa thèse, l'auteur poursuit trois objectifs interreliés. D'abord, il utilise le développement d'un marché mondial pour démontrer à quel point la construction ressemble beaucoup plus aux autres industries eu égard aux impératifs structuraux, aux contraintes d'accumulation de capital et à la concentration que la vision traditionnelle le reconnaît. Ensuite, l'auteur vise à démontrer le rôle concret des entreprises de construction dans la production et l'implantation de capital fixe par les autres industries à l'extérieur des États-Unis. Finalement, l'auteur vise à présenter une perspective historique de la transformation physique et de la reconstruction du Tiers Monde dues au transfert de technologie, d'ingénierie de construction en provenance des pays industrialisés dans le contexte d'une économie mondiale de marché orientée vers l'exploitation des richesses naturelles.

Pour atteindre ses objectifs, l'auteur divise son livre en trois parties. La première présente le cadre théorique utilisé et s'attarde à l'applicabilité à la construction de la distinction, commune aux autres industries, entre les exportations et l'investissement direct à l'étranger. La seconde partie présente l'histoire des activités transnationales de construction, d'entreprises américaines et européennes avant la Seconde Guerre, remontant même jusqu'à la construction du chemin de fer britannique dans les années 1830. La troisième partie est consacrée à l'évolution d'un marché mondial pour la construction.

Linder présente ici une facette de l'industrie de la construction que nous avons trop facilement tendance à oublier. Son analyse de la préfabrication dans tous les secteurs de la construction est fort instructive. Elle a le mérite de jeter une lumière claire sur un phénomène que d'aucuns ne voient pas ou ne veulent pas voir, comme cela fut d'ailleurs le cas pour les changements technologiques dans l'industrie de la construction. 
Cet ouvrage a cependant d'importantes limites. D'abord, vu le sujet traité, on y touche surtout à un seul secteur de la construction, celui des grands travaux. Et cela est normal. Mais l'analyse est présentée comme si l'industrie de la construction était homogène. Cela détonne surtout lorsque Linder traite de la préfabrication. Cet aspect aurait certes mérité un traitement plus approfondi et surtout plus nuancé eu égard aux différents secteurs de la construction.

Ensuite, l'aspect "relations du travail " de l'internationalisation de la construction est à peu près négligé sauf en ce qui a trait à l'attitude antisyndicale des multinationales américaines de construction. Les conditions de travail et les coûts de main-d'œuvre deviennent cruciaux pour comprendre cette internationalisation, d'autant plus qu'avec la pré- fabrication ou l'usinage, on construit de moins en moins. Plutôt on assemble.

Finalement, cet ouvrage souffre d'un défaut majeur. Linder ne présente aucune conclusion. $\mathrm{Vu}$ la perspective historique utilisée et vu l'éventail très large des expériences présentées, une bonne synthèse, une analyse approfondie et une vision de l'impact de ce phénomène de l'internationalisation de la construction sur les politiques publiques auraient été fort utiles.

Au-delà de ces limites, il demeure que cet ouvrage de Linder est utile, original et a le mérite de lever le voile sur un aspect trop souvent oublié, volontairement ou non, de l'industrie de la construction.

JEAN SEXTON

Université Laval

\section{Le Québec en jeu: comprendre les grands défis}

sous la direction de Gérard DAIGLE avec la collaboration de Guy ROCHER, Montréal, Presses de l'Université de Montréal, 1992, 811 p., ISBN 2-76061594-4.

Suite à la recension de cet ouvrage, Gérard Daible et Guy Rocher nous ont fait parvenir les précisions suivantes:

Nous avons pris connaissance de la recension de notre ouvrage Le Québec en jeu: comprendre les grands défis qu'en a faite Bernard Solasse dans la livraison du volume 49, numéro 3, 1994.

Il est bien évident que nous ne contestons d'aucune manière la liberté de monsieur Solasse d'exprimer son opinion personnelle sur cet ouvrage, tout comme nous avons le droit d'avoir aussi notre opinion sur la qualité de son compte rendu. Permettez-nous tout au moins de nous étonner que, dans une revue consacrée aux "relations industrielles ", l'auteur de cette recension n'ait pas dit un seul mot des deux chapitres portant expressément sur le champ d'intérêt de votre revue: le chapitre 4 de
Jacques Boucher sur les syndicats et le chapitre 5 de Gérard Hébert sur la négociation collective.

ll est cependant un point que nous ne pouvons laisser passer.

Monsieur Solasse affirme: "Avec la collaboration de Guy Rocher". Cette collaboration se résume apparamment (sic!) et vraisemblablement au seul "chapitre 15 "... soit vingthuit pages... ".

Cela signifie en clair que Gérard Daible a utilisé abusivement le nom de Guy Rocher, que celui-ci s'est prêté à cette manœuvre et que les Presses de l'Université de Montréal y ont consenti. Faire une telle affirmation sans aller aux sources - ce qui aurait été bien facile pour un chercheur! - relève d'une grande légèreté de jugement et d'un sérieux manque de probité intellectuelle. 